\title{
A Case Report of Recurrent Endometrial Stromal Sarcoma to the Breast, Lung and Pelvis after 15 years of Subtotal Hysterectomy
}

\author{
Shaimaa Shamoun* \\ Department of Clinical Oncology and Radiation Therapy, Jordan
}

*Corresponding author: Shaimaa Shamoun, Department of Clinical Oncology and Radiation Therapy, Clinical Oncology Nurse

Specialist, Al-Bashir Hospital, Al-Ashrafiyah, Amman, Jordan

\section{ARTICLE INFO}

Received: 慧 March 03, 2019

Published: 蔧 March 26, 2019

Citation: Shaimaa Shamoun. A Case Report of Recurrent Endometrial Stromal Sarcoma to the Breast, Lung and Pelvis after 15 years of Subtotal Hysterectomy. Biomed J Sci \& Tech Res 16(3)2019. BJSTR. MS.ID.002846.

Keywords: Low Grade Endometrial Sarcoma; Megace; Tamoxefin; Metastatis

\begin{abstract}
\section{Objective:}

a. To compare the effectiveness of tamoxifin and megestrol acetate drug on metastasis and recurrence low grade endometrial stromal sarcoma (LGESS).

b. To identify the interval between the first presentation of LGESS and the recurrence of (LGESS).

Methods: This is a rare case report of A 45-year-old woman with (LG-ESS), patients' history was collected by the authors ourselves through a structured interview, data was obtained from the patient's file including radiological pictures, pathological reports and drug doses. using PubMed data base for discussion this case. This woman with a history of hysteracomy and bilateral salpingooopharectomy 15 year earlier was admitted to $\mathrm{Al}$ Basheer hospital in 2015, presented with left breast mass, biopsy revealed (LGESS). CT scan have shown multiple breast and lung nodules. Doctor prescribed tamoxifen tab $20 \mathrm{mg}$ once daily. After three months of follow up, the size of the metastatic nodules was increased, new umbilical mass and left hemipelvic mass were discovered. Then, megestrol acetate oral suspension had been prescribed 160mg daily. After 6 months of follow up the patient shown good response to treatment; the size of metastatic lungs and breast nodules were decreased, Umbilical and left hemipelvic lesions were decrease, but new small focal liver lesion was seen.
\end{abstract}

Results: patient LG-ESS progression with tamoxifen by increasing the size of all nodules, but good response with megestrol acetate by decreasing the size of nodules. The disease was metastatic after 15 years old from first presentation.

Conclusion: Megestrol acetate was effective treatment rather than tamoxefin in metastatic case, so administration of megestrol acetate is recommended for metastasis and recurrence (LG-ESS).

\section{Highlights:}

a. Megestrol acetate was considered as effective hormonal therapy for LGSS recurrence, metastasis and non resectable by tumor size regression.

b. Tamoxifen was showed ineffective evidence by tumor size progression.

c. There is a possibility of recurrence of LGESS after long period of the first diagnosis (15) years in this case.

\section{Introduction}

Endometrial stromal sarcoma (ESS) is an extremely rare malignant tumor of the endometrium accounting for only $0.2 \%$ of all uterine malignancies and for $15-26 \%$ of primary uterine sarcomas. The annual incidence of ESS is 1-2 per million women [1], occurring 
in the age group of 40-50 years [1]. Histologically; ESS divided into low-grade and high-grade tumors. Low-grade endometrial stromal sarcoma (EES) is a rare tumor with a high recurrence rate but a favorable prognosis [2]. Relating to the treatment of ESS; Based on the literature review, it shows that low-grade ESS arising in the vagina can be treated by surgical resection effectively with close observation for recurrence or metastatic [3]. In addition, responses to adjuvant hormonal treatment of these recurrences and metastasis have been published in many case reports and series. We reporting a rare case of an endometrial stromal sarcoma metastasizing to the breast, liver, pelvis and lung after a 15-yearperiod in a 45 years old women.

\section{Case Study}

A 45 years old female as a case of metastatic breast sarcoma at 2015, have two girls and one boy, she aborted one time. Since about 17 years old, patient suffered from heavy vaginal bleeding for sex months duration and seeking health care provider to treat it. Patient was diagnosed by physician as a case of endometrial hyperplasia and fiboid, after that she did hysrtecomy and bilateral salpingo-oorphectomy then specimen was taken as a biopsy to confirm the diagnosis, but unfortunately she loose the pathological report. From 2000 until 2015 the patient didn't suffer from any sign and symptoms related to her illness. In October 2015 the patient felt accidentally hard irregular lump in her left breast associated with red color skin change. After seeking health care provider, the patient did complete breast mass excision, a pathological report showed metastatic low grade sarcoma of endometrial origin. This result was confirmed by pathological features and IHC staining in which CD10 and vimentin were positive, but desmin, S100, SMA and CD 34 were negative. PR and ER were positive and Her 2 neu was negative. CT scan examination had shown multiple lung nodules, largest one measuring $(2.9 * 2.9) \mathrm{cm}$ in the right lower lobe.

Others scattered over both lungs and 2 mass lesions was seen in the left breast $\left(2.3^{*} 2\right) \mathrm{cm}$ and $\left(3.6^{*} 2.5\right) \mathrm{cm}$. about $\left(7^{*} 6\right) \mathrm{cm}$ and $(1.4 * 1.2) \mathrm{cm}$ mass lesions seen in LOQ of the right breast. In November 2015 tamoxifen tab $20 \mathrm{mg}$ daily had been prescribed with follow up. In January 2016 abdominal ultrasound was done and it shown left kidney multiple cortical cystic lesions, the largest one about $5.5 \mathrm{~cm}$. follow up bilateral breast ultrasound was done, which was showed that there was large irregular in homogeneous measurement about(18*31) $\mathrm{mm}$ with internal calcification that was seen at 4 o'clock of left breast and right breast lesion with extended at lower outer at 8 o'clock which may represent with focal ductactisia.

In 1/3/2016 CAP -CT revealed multiple mass lesions variable in size, the largest about $3.6 * 3.9 \mathrm{~cm}$ in right lower lung lobe which represent secondary deposits. Multiple breast mass, the largest one in left side $\left(3.5^{*} 3.3\right) \mathrm{cm}$ with irregular outline and multiple small in right breast, the largest one about $1.2 \mathrm{~cm}$, few para aortic L.N.E, the largest about $1.7 \mathrm{~cm}$. left kidney showed severe hydronephrotic changes, loss of cortex a part from small area in upper pole with cortical thickness up to $0.5 \mathrm{~cm}$ with dilated ureters with no obvious cause. Umbilical mass about (4.2*4.2) which was mostly represent metastasis. Area of soft tissue density mass lesion seen in left hemipelvis about $4.8 * 5.3 \mathrm{~cm}$ with lobulated outline and some irregularity with areas of surrounding fat flames, which may represent matted L.N.E or recurrence. Kidney was severely hydronephrotic. After these CT scan results, the doctor decided to transform the patient from Tamoxifen $20 \mathrm{mg}$ to megestrol acetate (Megace ${ }^{\circledR}$ ) oral suspension $40 \mathrm{mg} / \mathrm{ml}$ (qid) with a total dose of $160 \mathrm{mg}$ daily associated with radiological follow up. In April 2016, Pelvic MRI was done, and it revealed a large $5.8 * 4.9 \mathrm{~mm}$ mass lesion at left side of the vaginal fornix, surrounding the distal left ureter and causing ureteric outflow obstruction. Uterus and both ovaries are not seen, MRI data are consistent with tumoral residue or recurrence within normal signal intensity bony and soft tissue structure.

In Jan. 2017 Renal scan was done to evaluate the function of the left kidney, which was revealed left kidney appears small in size and non-functional but right kidney normal with 100\% divided renal function and was not obstructed. In march 2017 and after about one year of Megace using, MRI pelvic exam was done and it was showed unchanged small less than $1 \mathrm{~cm}$ bilateral inguinal and pelvic L.N, no evidence of pelvic ascites, no definite bony destruction. So, impression of this MRI was no significant interval changes. In April 2017 follow up with CAP-CT scan with contrast was done; comparison was made to previous scan dated 17/11/2016. Which was showed decreased in amount of pericardial effusion, there were multiple variable size bilateral pulmonary nodules and few bilateral breast nodules were appearance smaller in size compared to previous scan. A hypo-dense liver lesion that measures $1 \mathrm{~cm}$ was not visualizing in the previous scan. In addition the nodules around the umbilical area and left hemipelvis mass were smaller in size compared to previous scan. The results of CT scan showed an interval regression in size of breast lesion, mediastinal L.N, lung deposits, umbilical deposits and left pelvic L.N .New small focal liver lesion which needs follow up rule out new metastasis. Last CAP-CT scan was done in July 2017 and it had shown an increased in amount of pericardial effusion in comparison to April exam with no other significant interval changes.

\section{Discussion}

Endometrial stromal sarcoma (ESS) is an uncommon neoplasm with the literature showing incidence of about $0.2 \%$ of all uterine malignancies and $10-15 \%$ of all uterine malignancies [4]. According to WHO classification Histologically; ESS divided into low-grade and high-grade tumors. Low grade ESS is common in middle aged females with mean age of 39 years and with better prognosis comparing with high grade ESS which was seen in older age female with a mean age of 61 years with aggressive behaviors and bad prognosis [2]. the 10 years overall survival is $65-76 \%$ in low grade ESS [5] in our case patient's age was 30 years old, premenopausal 
women when diagnosed LGSS that approximately consist with majority of cases in literature review [2] and the disease was metastatic after 15 years old .To support the ideas of LGSS has good prognosis comparing with high grade ESS; a A retrospective study was conducted in 2017 contain 38 patients with ESS (LGSS 28 patients \& HGSS 10Patients ),89\% and 62.9\% which were revealed overall survivor and 5 years disease free survival(DFS) respectively. And applying surgery in both HGESS and LGESS with adjuvant treatment were associated with improved 5-year DFS of $90.9 \%$ ( $<<$ 0.001) [6]. Another A retrospective review was conducted in 2009 confirm that LGESS was better prognosis comparing with HG-ESS which was yielded 30 patients with ESS in general (20 low-grade, 10 high-grade) who underwent surgical resection (hysterectomy, pelvic (P) and paraaortic (PA) lymphadenectomy),this review revealed $65 \%$ of patients have 5 years overall survival, with LGSS $79 \%$ versus HGSS $40 \%$ overall survival with $p=0.03$ [7-12].

In this case, the signs and symptoms of recurrence was happened after 15 years from initial problem that was treated by subtotal hysrtecomy and bilateral oorphectomy which was the main surgical procedures to treat LGSS and consider the first line treatment of LGSS [13]. Comparing to other case reports and series of LGESS, the disease may recur in a wide spectrum time ranged more than 17 years to less than 10 years. In a case report that was published by Khalifa MA et al in 1996 which was describing a 28-year-old women had LGESS with tumor recurred involved right ovary, sigmoid colon, small bowel, abdominal wall and omentum after 17 years [8]. Another case report of a 36-year-old woman with G1 LGESS, after twelve years later, the tumor recurred involved her pelvic and her peritoneal area [9]. In addition, the literature review of LGESS was published in 1991 which was presented 91 patients with initial disease confined to the uterus, 37 (41\%) developed recurrence within 10 years [10]. Patients age at diagnosis and their menstrual status were variable according to different literatures, one of these literatures was a Retrospective Review of Clinical Characteristics of Uterine Sarcoma that was conducted in 2017 which included 302 patients with uterine sarcomas (81 endometrial stromal sarcomas).

Mean age at diagnosis was 55 years, and 59\% were postmenopausal. The majority of patients with uterine sarcomas were postmenopausal. And the oncologist should be intervene with $41 \%$ of patients with uterine sarcoma had a high preoperative index of suspicion [11]. IHC used to confirm the diagnosis of LGSS , which is specific and sensitive to distinguish between uterine cancers especially between LGSS and leiomyosarcoma , in LGSS; ER,PR receptors and CD 10 are positive as what was found in Park and his colleagues case series which contains 10 cases of Endometrial stromal sarcoma that have positive CD10 and estrogen receptor (ER) in lung nodules biopsy that confirm that LG ESS was metastatic to the lug due to the same of IHC of it [12]. which was compatible with IHC in our case that revealed a positive test for $\mathrm{CD} 10+$, vimentin, $\mathrm{PR}$ and ER while Her 2 neu, desmin, S100, SMA and CD 34. Concerning to recurrence treatment, Physician prescribed tamoxifen tab. $20 \mathrm{mg}$ per day, after 3 months patients in our case did not show response with disease progression seen as increase in size of breast and lung nodules and appearance of another mass lesion which was seen in left hemipelvic and measured about $4.8 * 5.3 \mathrm{~cm}$.

In Reich and Regauer case series was conducted in 2006, that contains of 10 patients with endometrial stromal sarcomas (ESS) were on estrogen replacement therapy (ERT) or on tamoxifen. Physician was decided to discontinue of tamoxifen due to $8 / 10$ of patients were developed metastatic disease [14] disease progression with tamoxifen uses also seen in a case of a 32-year-old woman with FIGO stage II ESS using tamoxifen and local perfusion with cisplatin as a first line treatment resulted in disease progression and were discontinued. After that applying second therapy of treatment; using two cycles of combination chemotherapy with doxorubicin and ifosfamide followed by surgery resulted in complete remission after 5 years old [15]. On behalf of Sarcoma unit of the Royal Marsden NHS Foundation Trust. Professor Judson and Dr. Eirini Thanopoulou request the members of the NCCN Guidelines Panel for Uterine Neoplasms that conducted in 2012 to remove tamoxifen from the hormone therapy list for ESS due to negative impact of the use of tamoxifen in ESS (especially those, which are ER positive). Which has an agonist effect on endometrial stromal cells that stimulate tumor growth and increase in the incidence of uterine sarcomas in women taking tamoxifen.

Moreover, tamoxifen has been associated with earlier recurrence and disease progression in ESS that available in literature review [16]. Megace syrup $160 \mathrm{mg} /$ day was used as second choice after tamoxifen in our case which showed good response as tumor size regression. In three cases reports were reported in 2010 were mimic to our case ,those LG-ESS with lung and pelvic metastasis and have a history of hysterectomy, bilateral salpingo-oophorectomy ,all of three cases were treated with megestrol acetate for long periods and the disease under control from recurrence or local and distal metastatic [17] so surgery in addition using hormonal therapy for a long period achieve stabilization of the disease. Another case of LGESS was reported by Zaza KJ et al in 2015 with past history of uterine fibroids and treated by subtotal hysterectomy and left salpingo-oophorectomy ten years previously then treated by megestrol acetate, the disease was stable without any recurrence or metastatic for 2 years follow up [18].

Megestrol acetate was considered as effective hormonal therapy for multiple pulmonary metastases from LGSS which was prescribed in 2010 to the 30 years old female had LGSS with multiple pulmonary metastases and underwent Hysterectomy, right salpingo-oophorectomy, paraaortic and pelvic lymph node sampling, and omentectomy. Patient was treated with high dose megestrol acetate ( $800 \mathrm{mg} /$ day) for 4 months followed by $200 \mathrm{mg} /$ day for 20 months. The nodules began to regress and completely disappeared 17 months after surgery. Patient still alive with asymptomatic pulmo- 
nary metastases over 5 years with $200 \mathrm{mg}$ megestrol acetate daily [19]. In 2009, A case series contain 11patients with residual or recurrent LGESS treated by hormonal therapy just, were retrieved from patients' file with high response rate (median $48+$ months). (4 complete response; 5 partial response), one showed stable disease $(26+$ months $)$ and one progressive disease. This series confirm that hormonal therapy is a first line of treatment when LGSS recurrence and non resectable [20].

\section{Conclusion}

There is a possibility of recurrence of LGESS after long time of the first diagnosis (15) years in our case. Administration of adjuvant megestrol acetate daily was effective treatment to achieve favorable prognosis rather than administration of tamoxefin.

\section{Acknowledgment}

I would like to thank the patient himself for her participation and corporation to discuss this case study, she supported my work and helped me by answering for all question about her status with cooperative manner.

\section{Conflicts of Interest}

Conflict of interest relevant to this case study was not reported.

\section{References}

1. Puliyath G, Nair VR, Singh S (2010) Endometrial stromal sarcoma. Indian Journal of Medical and Paediatric Oncology : Official Journal of Indian Society of Medical \& Paediatric Oncology 31(1): 21-23.

2. Koyama T, Togashi K, Konishi I, Kobayashi H, Ueda H, et al. (1999) MR imaging of endometrial stromal sarcoma: correlation with pathologic findings. AJR Am J Roentgenol 173(3): 767-772.

3. Rivard C, Carter JS, Downs LS Jr (2015) Low-grade Endometrial Stromal Sarcoma Primarily Arising in the Vagina: A Case Report. J Reprod Med 60(9-10): 433-435.

4. Santos P, Cunha TM (2015) Uterine sarcomas: clinical presentation and MRI features. Diagn Interv Radiol 21(1): 4-9.

5. Chew I, Oliva E (2010) Endometrial Stromal Sarcomas. Adv Anat Pathol 17(2): 113-120.

6. Reshu A, Anupama R, Indu R (2017) Endometrial stromal sarcoma-A retropsective analysis of factors affecting recurrence. European journal of Obstetrics and gynecology and Reproductive Biopsy 21(6): 92-97.

7. Thomas MB, Keeney GL, Podratz KC (2009) Endometrial stromal sarcoma: treatment and patterns of recurrence. Int J Gynecol Cancer 19(2): 253-256.

\section{ISSN: 2574-1241}

\section{DOI: 10.26717/BJSTR.2019.16.002846}

Shaimaa Shamoun. Biomed J Sci \& Tech Res

This work is licensed under Creative Commons Attribution 4.0 License

Submission Link: https://biomedres.us/submit-manuscript.php
8. Khalifa MA, Hansen CH, Moore JL Jr, Rusnock EJ, Lage JM (1996) Endometrial stromal sarcoma with focal smooth muscle differentiation: recurrence after 17 years: a follow-up report with discussion of the nomenclature. Int J Gynecol Pathol 15(2): 171-176.

9. Krauss K, Bachmann C, Hartmann JT (2007) Management of late recurrence of a low-grade endometrial stromal sarcoma (LGESS): treatment with letrozole. Anticancer Res 27(5B): 3477-3480.

10. Bohr L, Thomsen CF (1991) Low-grade stromal sarcoma: a benign appearing malignant uterine tumour; a review of current literature. Differential diagnostic problems illustrated by four cases. Eur J Obstet Gynecol Reprod Biol 39(1): 63-69.

11. Wais M, Tepperman E, Bernardini MQ Gien LT, Jimenez W, et al. (2017) A Multicentre Retrospective Review of Clinical Characteristics of Uterine Sarcoma. J Obstet Gynaecol Can 39(8): 652-658.

12. Park JY, Sung CO, Jang SJ, Song SY, Han JH, et al. (2013) Pulmonary metastatic nodules of uterine low-grade endometrial stromal sarcoma: histopathological and immunohistochemical analysis of 10 cases. Histopathology 63(6): 833-840.

13. Cui R, Yuan F, Wang Y (2017) Clinicopathological characteristic and treatment strategies for Low grade endometrial stromal sarcoma. Medicine 96(15): e6584.

14. Reich O, Regauer S (2006) Estrogen replacement therapy and tamoxifen are contraindicated in patients with endometrial stromal sarcoma. Gynecol Oncol 102(2): 413-414.

15. Ihnen M, Mahner S, Jänicke F, Schwarz J (2007) Current treatment options in uterine endometrial stromal sarcoma: report of a case and review of the literature. nt J Gynecol Cancer 17(5): 957-963.

16. Judson, Eirini Thanopoulou (2012) Request of the members of the NCCN Guidelines Panel for Uterine Neoplasms to review the enclosed data for hormone therapy of uterine sarcomas. Sarcoma Unit Royal Marsden Hospital date of request.

17. Garavaglia E, Pella F, Montoli S (2010) Treatment of recurrent or metastatic low-grade endometrial stromal sarcoma: three case reports. Int J Gynecol Cancer 20(7): 1197-200.

18. Zaza KJ, Arafah MA, Al-Badawi IA (2015) Vulvar extrauterine endometrial stromal sarcoma: A case report and literature review.Hematol Oncol Stem Cell Ther 8(3): 125-129.

19. Lim MC, Lee S, Seo SS (2010) Megestrol acetate therapy for advanced low-grade endometrial stromal sarcoma. Epub 33(5): 260-262.

20. Dahhan T, Fons G, Buist MR, Ten Kate FJ, Van Der Velden J (2009) The efficacy of hormonal treatment for residual or recurrent low-grade endometrial stromal sarcoma. A retrospective study. Eur J Obstet Gynecol Reprod Biol 144(1): 80-84.

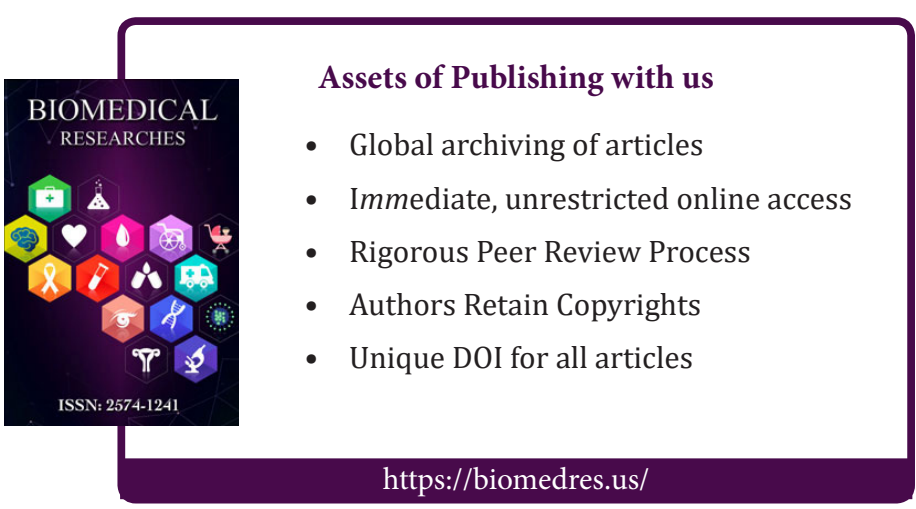

